\title{
On Logarithmic Order and Logarithmic Lower Order of Integral Functions
}

\author{
Aseel H. Abed Sadaa and Mustafa A. Sabri \\ Department of Mathematics, College of Basic Education, Mustansiriyah University, Baghdad, Iraq \\ mustafasabri.edbs@uomustansiriyah.edu.iq
}

\begin{abstract}
In this study, some relations between the logarithmic order and lower logarithmic order of integral functions of two complex variables are obtained in term of their Taylors series coefficients where the results have been given in the form of theorems.
\end{abstract}

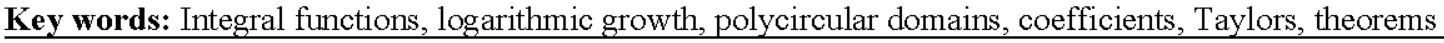

\section{INTRODUCTION}

A series of the form:

$$
f(s)=\sum_{n=1}^{\infty} a_{n} \exp \left(s \lambda_{n}\right) \text { is called integral Dirichlet series }
$$

where, $\mathrm{s}=\sigma+\mathrm{it}\left(\boldsymbol{\sigma}\right.$, treal variables), a sequence $\left\{\mathrm{a}_{\mathrm{n}}\right\} \subseteq \mathrm{C}$ and $\left\{\lambda_{n}\right\}$ is a strictly increasing sequence of positive real numbers such that:

$$
0<\lambda_{1}<\lambda_{2}<, \ldots,<\lambda_{n} \rightarrow \infty,(n \rightarrow \infty) \text {, set } \lim _{n \rightarrow \infty} \frac{\sup _{n}}{\lambda_{n}}=D^{*}<\infty
$$

Let $\sigma_{\mathrm{c}}$ and $\sigma_{\alpha}$ be the abscissa of convergence and abscissa of absolute convergence of $\mathrm{f}(\mathrm{s})$, then, the following (Ritt, 1928) is satisfy:

$$
\begin{gathered}
0 \leq \sigma_{\mathrm{c}}-\sigma_{\alpha} \leq \mathrm{D}^{*} \\
\sigma_{\mathrm{c}}=-\lim _{n \rightarrow \infty} \frac{\sup \log \left|\mathrm{a}_{\mathrm{n}}\right|}{\lambda_{\mathrm{n}}}
\end{gathered}
$$

Thus, if $\mathrm{D}^{*}<\infty$ and $\sigma_{\mathrm{c}}=\infty, \mathrm{f}(\mathrm{s})$ represents an integral function and by Eq. $4 \sigma_{\alpha}=\infty$, so that, the series $f(s)=\sum_{n=1}^{\infty} a_{n} \exp \left(s \lambda_{n}\right)$ converges absolutely at every point of the finite complex plane. Further, for $\mathrm{D}^{*}=0$, we get:

$$
\sigma_{\alpha}=\sigma_{c}=-\lim _{n \rightarrow \infty} \sup \frac{\log \left|a_{n}\right|}{\lambda_{n}}
$$

Let, $\mathrm{M}(\sigma)$ defined as Izumi (1929):

$$
\mathrm{M}(\sigma)=\underset{\text { L. ustem }}{\text { L.u. }}|\mathrm{f}(\sigma+\mathrm{it})|
$$

and:

$$
\mathrm{u}(\sigma)=\max _{\mathrm{n} z 1}\left\{\left|\mathrm{a}_{\mathrm{n}}\right| \mathrm{e}^{\sigma \lambda_{\mathrm{n}}}\right\}, \text { also }
$$

$\mathrm{v}(\boldsymbol{\sigma})$ defined as Izumi (1929):

$$
\mathrm{v}(\sigma)=\max \left\{\mathrm{n}: \mathrm{u}(\sigma)=\left|\mathrm{a}_{\mathrm{n}}\right| \mathrm{e}^{\sigma \lambda_{\mathrm{n}}}\right\}
$$

As usual, if there are more than one maximum term, we take $\mathrm{v}(\sigma)$ to be the highest index. Several researchers have studied the properties of $\mathrm{M}(\sigma), \mathrm{u}(\sigma)$ and $\lambda_{\mathrm{v}(\sigma)}$. To study the growth properties of integral function $\mathrm{f}(\mathrm{s})$, definitions of order $\rho$ and lower order $\lambda$ where given by Ritt (1928) as:

$$
\begin{aligned}
& \rho=\lim _{\sigma \rightarrow \infty} \frac{\sup (\log \log \mathrm{M}(\sigma))}{\sigma} \\
& \lambda=\lim _{\sigma \rightarrow \infty} \frac{\inf (\log \log \mathrm{M}(\sigma))}{\sigma}
\end{aligned}
$$

\section{MATERIALS AND METHODS}

$$
\text { Let } f\left(z_{1}, z_{2}\right)=\sum_{m, n=0}^{\infty} a_{m, n} z_{1}^{\lambda_{m}} z_{2}^{\mu_{n}}
$$

$\mathrm{Be}$ a non-constant integral function where $0<\lambda_{1}<$ $\lambda_{2}<, \ldots, \lambda_{\mathrm{m}} \rightarrow \infty, 0<\mu_{1}<\mu_{2}<, \ldots,<\mu_{\mathrm{n}} \rightarrow \infty$ are strictly increasing sequence of positive integers such that no element of the sequence $\left\{a_{\mathrm{m}, n}\right\}_{\mathrm{m}, \mathrm{n}=1}^{\infty}$ is zero. The maximum modulus of integral function is denoted by Gol'dberg (1959):

$$
\mathrm{M}_{\mathrm{G}}(\mathrm{r}, \mathrm{f})=\max _{\left(2, z_{2}\right) \overline{\mathrm{F}}_{\mathrm{r}}}\left|\mathrm{f}\left(\mathrm{z}_{1}, \mathrm{z}_{2}\right)\right|
$$

The maximum term $u(r)$ of integral function $f\left(z_{1}, z_{2}\right)$ is defined as Gol'dberg (1959):

Corresponding Author: Mustafa A. Sabri, Department of Mathematics, College of Basic Education, 


$$
\mathrm{u}(\mathrm{r}, \mathrm{f})=\max _{(\mathrm{m}, \mathrm{n})>(0,0)}\left\{\left|\mathrm{a}_{\mathrm{m}, \mathrm{n}}\right| \mathrm{r}^{\left(\lambda_{\mathrm{m}}+\mu_{\mathrm{m}}\right)}\right\}
$$

where, $\overline{\mathrm{G}}_{x}$ be the family of closed polycircular domains in space $\left(z_{1}, z_{2}\right)$ dependent on parameter $r>0$ and possess the property that $\left(z_{1}, z_{2}\right) \in \overline{\mathrm{G}}_{x}$, if and only if $\left(z_{1} / r, z_{2} / r\right) \in \bar{G}_{r}$. For the integral function (Eq. 11), Gol'dberg (1959) obtained the order in terms of coefficients of its Taylor expansion as:

$$
\rho=\lim _{m, n \rightarrow \infty} \sup \frac{\log \left|\lambda_{m}+\mu_{n}\right|}{\log \left(\lambda_{m}+\mu_{n}\right)^{-1} \log \left|a_{m, n}\right|^{-1}}
$$

where, $0<\rho<\infty$. If the order $\rho=0$ then, the function (Eq. 11) is called of order zero (slow growth). The theory of integral function of slow growth has been enriched by the research of Iyer (1942). Later on, Shah and Singh (1958) derived formulae for logarithmic order in terms of coefficients of its Taylor expansion as:

$$
\rho^{*}-1=\limsup _{m, n \rightarrow \infty} \frac{\log \left|\lambda_{m}+\mu_{n}\right|}{\log \left(\left(\lambda_{m}+\mu_{n}\right)^{-1} \log \left|a_{m, n}\right|^{-1}\right)}
$$

A corresponding result for the lower logarithmic order $\lambda^{*}$ does not always hold. In fact, it has been shown that, if:

$$
\begin{gathered}
\log \left(\lambda_{\mathrm{m}}+\mu_{\mathrm{n}}\right) \sim \log \left(\lambda_{\mathrm{m}+1}+\mu_{\mathrm{n}+1}\right) \text {, then } \\
\liminf _{\mathrm{m}, \mathrm{n} \rightarrow \infty} \frac{\log \left|\lambda_{\mathrm{m}}+\mu_{\mathrm{n}}\right|}{\log \left(\left(\lambda_{\mathrm{m}}+\mu_{\mathrm{n}}\right)^{-1} \log \left|\mathrm{a}_{\mathrm{m}, \mathrm{n}}\right|^{-1}\right)} \leq \lambda^{*}-1
\end{gathered}
$$

But if Kumar and Rastogi (2014):

$$
\log \frac{\left|a_{m}, n\right|}{\left|a_{m+1, n+1}\right|^{-1}} /\left(\lambda_{m+1}-\lambda_{m}\right)\left(\mu_{n+1}-\mu_{n}\right)
$$

Forms a non-decreasing sequence function of $m, n$ for $\mathrm{m} \geq \mathrm{m}_{0}$ and $\mathrm{n} \geq \mathrm{n}_{0}$ then:

$$
\liminf _{m, n \rightarrow \infty} \frac{\log \left|\lambda_{m}+\mu_{n}\right|}{\log \left(\left(\lambda_{m}+\mu_{n}\right)^{-1} \log \left|a_{m, n}\right|^{-1}\right)} \geq \lambda^{*}-1
$$

If logarithmic order is equal to logarithmic lower order $\left(\rho^{*}=\lambda^{*}\right)$, then, the function Eq. (11) is said to be of regular growth. In this study, some formulae in terms of the coefficients of Taylor series for logarithmic order, lower logarithmic order have been obtained as theorems in the next section.

\section{RESULTS AND DISCUSSION}

\section{Theorem 3.1:}

$$
\begin{aligned}
\text { Let } f_{1}\left(z_{1}, z_{2}\right) & =\sum_{m, n=1}^{\infty} a_{m, n} z_{1}^{\lambda_{1, m}} z_{2}^{\mu_{1, n}} \\
f_{2}\left(z_{1}, z_{2}\right) & =\sum_{m, n=1}^{\infty} b_{m, n} z_{1}^{\lambda_{2, m}} z_{2}^{\mu_{2, n}}
\end{aligned}
$$

be integral functions of regular logarithmic growth such that:

$$
\begin{gathered}
\log \left(\lambda_{1, \mathrm{~m}}+\mu_{1, \mathrm{n}}\right) \sim \log \left(\lambda_{2, \mathrm{~m}}+\mu_{2, \mathrm{n}}\right) \sim \log \left(\lambda_{\mathrm{m}}+\mu_{\mathrm{n}}\right) \\
\log \left(\lambda_{\mathrm{m}}+\mu_{\mathrm{n}}\right) \sim \log \left(\lambda_{\mathrm{m}+1}+\mu_{\mathrm{n}+1}\right) \\
\log \frac{\left|\mathrm{a}_{\mathrm{m}, \mathrm{n}}\right|}{\left|\mathrm{a}_{\mathrm{m}+1, \mathrm{n}+1}\right|} /\left(\lambda_{1, \mathrm{~m}+1}+\lambda_{1, \mathrm{~m}}\right)\left(\mu_{1, \mathrm{n}+1}-\mu_{1, \mathrm{n}}\right) \\
\log \frac{\left|\mathrm{b}_{\mathrm{m}, \mathrm{n}}\right|}{\left|\mathrm{b}_{\mathrm{m}+1, \mathrm{n}+1}\right|} /\left(\lambda_{2, \mathrm{~m}+1}+\lambda_{2, \mathrm{~m}}\right)\left(\mu_{2, \mathrm{n}+1}-\mu_{2, \mathrm{n}}\right)
\end{gathered}
$$

be non-decreasing sequence function of $\mathrm{m}, \mathrm{n}$ for $\mathrm{m}, \mathrm{n}$ for $\mathrm{m} \geq \mathrm{m}_{0}$ and $\mathrm{n} \geq \mathrm{n}_{0}$. Then, $\mathrm{f}_{1}\left(\mathrm{z}_{1}, \mathrm{z}_{2}\right)$ and $\mathrm{f}_{2}\left(\mathrm{z}_{1}, \mathrm{z}_{2}\right)$ of same $\log$ arithmic order $\rho^{*}$, if and only if:

$$
\log \left\{\frac{\log \left|b_{m, n}\right|}{\log \left|a_{m, n}\right|}\right\}=o\left(\log \left(\lambda_{m}+\mu_{n}\right)\right), \text { as } m, n \rightarrow \infty
$$

Proof: Since, $f_{1}\left(z_{1}, z_{2}\right)$ and $f_{2}\left(z_{1}, z_{2}\right)$ is of regular logarithmic growth of logarithmic order $\rho^{*}$, it implies that from Eq. (15-17):

$$
\begin{gathered}
\limsup _{m, n \rightarrow \infty} \frac{\log \left|\lambda_{1, m}+\mu_{1, n}\right|}{\log \left(\left(\lambda_{1, m}+\mu_{1, n}\right)^{-1} \log \left|a_{m, n}\right|^{-1}\right)}=\rho^{*}-1= \\
\lambda^{*}-1=\liminf _{m, n \rightarrow \infty} \frac{\log \left|\lambda_{1, m}+\mu_{1, n}\right|}{\log \left(\left(\lambda_{1, m}+\mu_{1, n}\right)^{-1} \log \left|a_{m, n}\right|^{-1}\right)}
\end{gathered}
$$

Further, since, $\log \left(\lambda_{i, \mathrm{~m}}+\mu_{1, \mathrm{n}}\right) \sim \log \left(\lambda_{\mathrm{m}}+\mu_{\mathrm{n}}\right)$, this gives:

$$
\limsup _{m, n \rightarrow \infty} \frac{\log \left(\left(\lambda_{m}+\mu_{n}\right)^{-1} \log \left|a_{m, n}\right|^{-1}\right)}{\log \left|\lambda_{m}+\mu_{n}\right|}=\frac{1}{\rho^{*}-1}
$$

Similarly, for the function $\mathrm{f}_{2}\left(\mathrm{z}_{1}, \mathrm{z}_{2}\right)$, if it is of regular logarithmic growth of logarithmic order $\mathrm{p}^{*}$, it implies that:

$$
\limsup _{m, n \rightarrow \infty} \frac{\log \left(\left(\lambda_{m}+\mu_{n}\right)^{-1} \log \left|b_{m, n}\right|^{-1}\right)}{\log \left|\lambda_{m}+\mu_{n}\right|}=\frac{1}{\rho^{*}-1}
$$


Subtracting Eq. (21) from Eq. (22) this gives Eq. (11) again, if $\rho_{1}^{*}, \rho_{2}^{*}$ are the logarithmic orders of $f_{1}\left(z_{1}, z_{2}\right)$ and $f_{2}\left(z_{1}, z_{2}\right)$, respectively this gives:

$$
\frac{1}{\rho_{1}^{*}-1}-\frac{1}{\rho_{2}^{*}-1}=\frac{\log \left\{\frac{\log \mid b_{m, n}}{\log \mid a_{m, n}}\right\}}{\log \left|\lambda_{m}+\mu_{n}\right|}=0
$$

If Eq. (19) hold then $\rho_{1}^{*}-1, \rho_{2}^{*}-1$ :

Theorem 3.2: Let:

$$
\begin{aligned}
& \mathrm{f}_{1}\left(\mathrm{z}_{1}, \mathrm{z}_{2}\right)=\sum_{\mathrm{m}, \mathrm{n}=1}^{\infty} \mathrm{a}_{\mathrm{m}, \mathrm{n}} \mathrm{z}_{1}^{\lambda_{1, \mathrm{~m}}} \mathrm{z}_{2}^{\mu_{1, \mathrm{n}}} \\
& \mathrm{f}_{2}\left(\mathrm{z}_{1}, \mathrm{z}_{2}\right)=\sum_{\mathrm{m}, \mathrm{n}=1}^{\infty} \mathrm{b}_{\mathrm{m}, \mathrm{n}} \mathrm{z}_{1}^{\lambda_{2, \mathrm{~m}}} \mathrm{z}_{2}^{\mu_{2, \mathrm{n}}} \\
& \mathrm{f}_{3}\left(\mathrm{z}_{1}, \mathrm{z}_{2}\right)=\sum_{\mathrm{m}, \mathrm{n}=1}^{\infty} \mathrm{c}_{\mathrm{m}, \mathrm{n}} \mathrm{z}_{1}^{\lambda_{3, \mathrm{~m}}} \mathrm{z}_{2}^{\mu_{3, \mathrm{n}}}
\end{aligned}
$$

be integral functions of logarithmic orders and respectively such that, $\rho_{1}^{*}, \rho_{2}^{*}$ and $\rho_{3}^{*}$, respectively such that:

$$
\begin{gathered}
\log \left(\lambda_{1, \mathrm{~m}}+\mu_{1, \mathrm{n}}\right) \sim \log \left(\lambda_{2, \mathrm{~m}}+\mu_{2, \mathrm{n}}\right) \sim \\
\log \left(\lambda_{3, \mathrm{~m}}+\mu_{3, \mathrm{n}}\right) \sim \log \left(\lambda_{\mathrm{m}}+\mu_{\mathrm{n}}\right) \\
\sqrt{\log \left(\left(\lambda_{\mathrm{m}}+\mu_{\mathrm{n}}\right)^{-1} \log \left|\mathrm{a}_{\mathrm{m}, \mathrm{n}}\right|^{-1} \log \left(\left(\lambda_{\mathrm{m}}+\mu_{\mathrm{n}}\right)^{-1} \log \left|\mathrm{b}_{\mathrm{m}, \mathrm{n}}\right|^{-1}\right)\right.} \\
\log \left(\left(\lambda_{\mathrm{m}}+\mu_{\mathrm{n}}\right)^{-1} \log \left|\mathrm{c}_{\mathrm{m}, \mathrm{n}}\right|^{-1}\right)
\end{gathered}
$$

Then:

$$
\rho_{3}^{*}-1 \leq \sqrt{\left(\rho_{1}^{*}-1\right)\left(\rho_{2}^{*}-1\right)}
$$

Proof: From the definition of logarithmic orders $\rho_{1}^{*}, \rho_{2}^{*}$ and first condition Eq. (25) that:

$$
\limsup _{m, n \rightarrow \infty} \frac{\log \left(\left(\lambda_{m}+\mu_{n}\right)^{-1} \log \left|a_{m, n}\right|^{-1}\right)}{\log \left|\lambda_{m}+\mu_{n}\right|} \geq \frac{1}{\rho_{1}^{*}-1}-\frac{E}{2}
$$

and:

$$
\limsup _{m, n \rightarrow \infty} \frac{\log \left(\left(\lambda_{m}+\mu_{n}\right)^{-1} \log \left|b_{m, n}\right|^{-1}\right)}{\log \left|\lambda_{m}+\mu_{n}\right|} \geq \frac{1}{\rho_{2}^{*}-1}-\frac{E}{2}
$$

Now, from Eq. (28 and 29) and for arbitrarily $\epsilon$ it gives that:

$$
\begin{gathered}
\frac{\sqrt{\log \left(\left(\lambda_{\mathrm{m}}+\mu_{\mathrm{n}}\right)^{-1} \log \left|\mathrm{a}_{\mathrm{m}, \mathrm{n}}\right|^{-1} \log \left(\left(\lambda_{\mathrm{m}}+\mu_{\mathrm{n}}\right)^{-1} \log \left|\mathrm{b}_{\mathrm{m}, \mathrm{n}}\right|^{-1}\right)\right.}}{\log \left(\lambda_{\mathrm{m}}+\mu_{\mathrm{n}}\right)} \\
\sqrt{\frac{1}{\left(\rho_{1}^{*}-1\right)} \cdot \frac{1}{\left(\rho_{2}^{*}-1\right)}}
\end{gathered}
$$

Using condition (ii), to get:

or:

$$
\frac{\log \left(\left(\lambda_{\mathrm{m}}+\mu_{\mathrm{n}}\right)^{-1} \log \left|\mathrm{e}_{\mathrm{m}, \mathrm{n}}\right|^{-1}\right)}{\log \left(\lambda_{\mathrm{m}}+\mu_{\mathrm{n}}\right)} \geq \sqrt{\frac{1}{\left(\rho_{1}^{*}-1\right)} \cdot \frac{1}{\left(\rho_{2}^{*}-1\right)}}
$$

$$
\rho_{3}^{*}-1 \leq \sqrt{\left(\rho_{1}^{*}-1\right)\left(\rho_{2}^{*}-1\right)}
$$

Corollary: Let $f_{1}\left(z_{1}, z_{2}\right)=\sum_{m, n=1}^{\infty} a_{m, n}^{i} z_{1}^{\lambda_{1, n}} z_{2}^{\mu_{i n}}$ for $i=1,2, \ldots, p$ be integral functions of logarithmic orders $\rho_{1}^{*}, i=1,2, \ldots$, p such that:

$$
\begin{gathered}
\log \left(\lambda_{\mathrm{i}, \mathrm{m}}+\mu_{\mathrm{i}, \mathrm{n}}\right) \sim \log \left(\lambda_{\mathrm{m}}+\mu_{\mathrm{n}}\right), \text { for } \mathrm{i}=1,2, \ldots, \mathrm{p} \\
\sqrt{\left(\prod_{\mathrm{i}=1}^{\mathrm{p}} \log \left(\left(\lambda_{\mathrm{i}, \mathrm{m}}+\mu_{\mathrm{i}, \mathrm{n}}\right)^{-1} \log \left|\mathrm{a}_{\mathrm{m}, \mathrm{n}}^{\mathrm{i}}\right|^{-1}\right)\right.} \\
\log \left(\left(\lambda_{\mathrm{m}}+\mu_{\mathrm{n}}\right)^{-1} \log \left|\mathrm{a}_{\mathrm{m}, \mathrm{n}}\right|^{-1}, \text { for } \mathrm{i}=1,2, \ldots, \mathrm{p}\right.
\end{gathered}
$$

Then:

$$
\rho^{*}-1 \leq \sqrt{\prod_{i=1}^{p}\left(\rho_{i}^{*}-1\right)}
$$

where, $\rho^{*}$ is logarithmic order of the integral function $f\left(z_{1}, z_{2}\right)=\sum_{m, n=1}^{\infty} a_{m, n} z_{1}^{\lambda_{1, m}} z_{2}^{\mu_{1, n}}$.

\section{Theorem 3.3: Let:}

$$
\begin{aligned}
& \mathrm{f}_{1}\left(\mathrm{z}_{1}, \mathrm{z}_{2}\right)=\sum_{\mathrm{m}, \mathrm{n}=1}^{\infty} \mathrm{a}_{\mathrm{m}, \mathrm{n}} \mathrm{z}_{1}^{\lambda_{1, \mathrm{~m}}} \mathrm{z}_{2}^{\mu_{1, \mathrm{n}}} \\
& \mathrm{f}_{2}\left(\mathrm{z}_{1}, \mathrm{z}_{2}\right)=\sum_{\mathrm{m}, \mathrm{n}=1}^{\infty} \mathrm{b}_{\mathrm{m}, \mathrm{n}} \mathrm{z}_{1}^{\lambda_{2, \mathrm{~m}}} \mathrm{z}_{2}^{\mu_{2, \mathrm{n}}} \\
& \mathrm{f}_{3}\left(\mathrm{z}_{1}, \mathrm{z}_{2}\right)=\sum_{\mathrm{m}, \mathrm{n}=1}^{\infty} \mathrm{c}_{\mathrm{m}, \mathrm{n}} \mathrm{z}_{1}^{\lambda_{3, \mathrm{~m}}} \mathrm{z}_{2}^{\mu_{3, \mathrm{n}}}
\end{aligned}
$$

be integral functions of logarithmic orders and, respectively such that, $\rho_{1}^{*}, \rho_{2}^{*}$ and $\rho_{3}^{*}$, respectively such that:

$$
\begin{gathered}
\log \left(\lambda_{1, \mathrm{~m}}+\mu_{1, \mathrm{n}}\right) \sim \log \left(\lambda_{(2, \mathrm{~m})}+\mu_{(2, \mathrm{n})}\right) \sim \\
\log \left(\lambda_{3, \mathrm{~m}}+\mu_{3, \mathrm{n}}\right) \sim \log \left(\lambda_{\mathrm{m}}+\mu_{\mathrm{n}}\right) \\
\left.\log \left\{\log \left|\mathrm{a}_{(\mathrm{m}, \mathrm{n})}\left(\frac{1}{\left(\lambda_{\mathrm{m}}+\mathrm{H}_{\mathrm{n}}\right)}\right) \log \right| \mathrm{b}_{(\mathrm{m}, \mathrm{n})} \mid \frac{1}{\left(\lambda_{\mathrm{m}}+\mu_{\mathrm{m}}\right)}\right)\right\}\left.\sim \log (\log \mid \mathrm{c})_{\mathrm{m}, \mathrm{n}}\right|^{\frac{1}{\lambda_{\mathrm{m}}+\mu_{\mathrm{n}}}}
\end{gathered}
$$

Then:

$$
\rho_{3}^{*}-1 \leq\left(\rho_{1}^{*}-1\right)+\left(\rho_{2}^{*}-1\right)
$$

Proof: From the definition of logarithmic orders $\rho_{1}^{*}, \rho_{2}^{*}$ and first condition (Eq. 29): 


$$
\lim _{m, n \rightarrow \infty} \sup \frac{\log \left(\log \left|a_{m, n}\right|^{\frac{1}{\lambda_{m}+\mu_{n}}}\right)}{\log \left|\lambda_{m}+\mu_{n}\right|} \geq \frac{1}{\rho_{1}^{*}-1}-\frac{E}{2}
$$

and:

$$
\lim _{m, n \rightarrow \infty} \sup \frac{\log \left(\log \left|b_{m, n}\right|^{\frac{1}{\lambda_{m}+\mu_{n}}}\right)}{\log \left|\lambda_{m}+\mu_{n}\right|} \geq \frac{1}{\rho_{2}^{*}-1}-\frac{E}{2}
$$

Summation (Eq. 35) and (36), it gives that:

or:

$$
\frac{\log \left(\log \left|\mathrm{a}_{\mathrm{m}, \mathrm{n}}\right|-\frac{1}{\lambda_{\mathrm{m}}+\mu_{\mathrm{n}}}\right)+\log \left(\log \left|\mathrm{b}_{\mathrm{m}, \mathrm{n}}\right|^{\frac{1}{\lambda_{\mathrm{m}}+\mu_{\mathrm{n}}}}\right)}{\log \left|\lambda_{\mathrm{m}}+\mu_{\mathrm{n}}\right|} \geq
$$

$$
\begin{aligned}
& \log \left|\lambda_{\mathrm{m}}+\mu_{\mathrm{n}}\right| \\
& \frac{1}{\rho_{2}^{*}-1}+\frac{1}{\rho_{2}^{*}-1}-\in
\end{aligned}
$$

Now using condition (Eq. 30) and for arbitrarily $\epsilon$, to get:

or:

$$
\frac{\log \left(\log \left|\mathrm{c}_{\mathrm{m}, \mathrm{n}}\right|^{\frac{1}{\lambda_{\mathrm{m}}+\mu_{\mathrm{n}}}}\right)}{\log \left|\lambda_{\mathrm{m}}+\mu_{\mathrm{n}}\right|} \geq \frac{1}{\rho_{1}^{*}-1}+\frac{1}{\rho_{2}^{*}-1}
$$

$$
\rho_{3}^{*}-1 \leq\left(\rho_{1}^{*}-1\right)+\left(\rho_{2}^{*}-1\right)
$$

\section{CONCLUSION}

In this study, we have some inequality in term of order and lower order of multiple Dirichlet series of slow growth by using some conditions.

\section{ACKNOWLEDGEMENT}

The researchers would like to thank Mustansiriyah University (www.uomustansiryah.edu.iq) Baghdad, Iraq for its support in the present research.

\section{REFERENCES}

Gol'dberg, A.A., 1959. Elementary remarks on the formulas defining order and type of functions of several variable. Akad. Nauk. Armjan SSR. Dokl., 29: 145-151.

Iyer, V.G., 1942. A property of the maximum modulus of integral functions. J. Indian Math. Soc., 6: 69-80.

Izumi, S.I. 1929. Integral functions defined by Dirichlet's series. Jpn. J. Math. Trans. Abstracts, 6: 199-204.

Kumar, A. and A. Rastogi, 2014. On the order and type of entire functions represented by dirichlet series in two variables. J. Global Res. Math. Arch., 2: 37-42.

Ritt, J.F., 1928. On certain points in the theory of Dirichlet series. Am. J. Math., 50: 73-86.

Shah, S.M. and S.K. Singh, 1958. Note on a step function. Q. J. Math., 9: 63-67. 